\title{
Non-alcoholic steatohepatitis or metabolic-associated fatty liver: time to change
}

\author{
Gamal Shiha $^{1,2}$, Nasser Mousa ${ }^{3}$ \\ ${ }^{1}$ Egyptian Liver Research Institute and Hospital (ELRIAH), Mansoura, Egypt; ${ }^{2}$ Hepatology and Gastroenterology Unit, Internal Medicine \\ Department, ${ }^{3}$ Tropical Medicine Department, Faculty of Medicine, Mansoura University, Mansoura, Egypt \\ Correspondence to: Gamal Shiha. Internal Medicine Department, Faculty of Medicine, Mansoura University, Mansoura, Egypt; Egyptian Liver \\ Research Institute and Hospital (ELRIAH), Mansoura, Egypt. Email: g_shiha@hotmail.com. \\ Comment on: Eslam M, Sanyal AJ, George J, et al. MAFLD: a consensus-driven proposed nomenclature for metabolic associated fatty liver disease. \\ Gastroenterology 2020;158:1999-2014.e1.
}

Submitted Apr 20, 2020. Accepted for publication Jun 12, 2020.

doi: $10.21037 / \mathrm{hbsn}-20-438$

View this article at: http://dx.doi.org/10.21037/hbsn-20-438

Non-alcoholic fatty liver disease (NAFLD) is a range of liver disease characterized by the occurrence of steatosis in greater than $5 \%$ of hepatocytes of people who drink tiny or no alcohol. NAFLD comprises, a benign nonalcoholic fatty liver (NAFL), and the more serious nonalcoholic steatohepatitis (NASH) described by steatosis, hepatocellular ballooning, lobular inflammation and lastly cirrhosis (1).

NAFL and NASH are still negative defining; affected person is categorized as having NAFL/NASH when the amount of alcohol intake /day is lesser than 20-30 g (140 g/week), and all different etiologies of liver diseases (hepatitis $\mathrm{C}$ virus, hepatitis $\mathrm{B}$ virus, drug-induced, autoimmune liver disease, etc.) have been excluded. As suggested newly, it is perhaps the time to reach an overall consensus throughout all the scientific community to revolution the nomenclature, and go from a negative to a positive definition of NAFLD/ NASH (2).

In the Gastroenterology, Eslam and colleagues suggested that the heterogeneous pathogenesis of metabolic fatty liver diseases and incorrectness in terminology and definitions require a revision of nomenclature to notify scientific trial design and drug development. They advised that, considering NAFLD heterogeneity in clinical practice has many treasured medical affects (3).

On behalf of this international consensus panel (3) a provided suggestion on terminology that more accurately reflects pathogenesis is "metabolic-associated fatty liver" (MAFLD). This new nomenclature can help in patient stratification for management. Moreover, expert panel reached consensus that, NAFLD/NASH acronyms, the methods for diagnosis and a deficiency of adequate thought of heterogeneity in risk population and treatment responsiveness are out-of-date and make barriers that delay discover of high-quality therapy. The consensus group believes that, the acronym MAFLD is more correctly reveals existing knowledge of fatty liver diseases linked with metabolic dysfunction and should replace the current NAFLD/NASH as it will push the efforts of research community to update the nomenclature and subphenotype the disease to speed the translational path to new treatments.

The view of the consensus to change the term NAFLD to MAFLD based on four aspects. First, NAFLD was diagnosed as a disorder of exclusion, which needs, exclusions of other chronic liver diseases for example, viral hepatitis, autoimmune diseases or alcohol fatty liver disease. Though, with advances in thoughtful of the principal pathological processes of NAFLD, it is clearly a disease that must be defined by inclusion, instead than exclusion. Additional, fatty liver disease is known to coincide with other diseases, for example, viral hepatitis, autoimmune diseases, consuming a westernized diet and alcohol (4), and accelerate the disease progression (5). Therefore, the consensus advises that, the terminology for fatty liver disease and standards for diagnosis require to mirror this new information.

Second, is the problem of linking alcohol intake to 
metabolic fatty liver disease in its name that is a distinct entity to alcohol. Furthermore, involving the term "nonalcoholic" in the name is unacceptable for person who stop drinking. Also, the debate about the safety amount of alcohol intake is a raising question. Notably, there is a critical requisite to recognize simultaneous metabolic and alcohol liver disease, therefore they may be treated correctly.

Third, the debate about the clinical classification of patients into NASH patients and those without and whether this is the appropriate classification. The consensus suggests the present classification may be confusing and possibly metabolic dysfunction linked fatty liver disease should be considered similar to other chronic liver diseases with various degree of activity-stage of fibrosis, lacking dichotomous stratification into NASH and non-NASH.

Fourth, the consensus recommendations are against considering or managing fatty liver diseases as a single condition with a single shot hitting multiple targets because the heterogeneous nature of fatty liver diseases proposes that, they cannot be thought of or treated as a single disorder. Moreover, they suggested that, negligence of heterogeneity influences and diminishes the capacity to exactly define the natural history of fatty liver phenotypes, to correctly choice for clinical trials.

This consensus is a pivotal step in the development of more accurate nomenclature for MAFLD that reflecting the NAFLD heterogeneity and can help in patient stratification for better clinical trials strategy and the capacity to find successful management.

This consensus has many strength points include, first, highlight the importance of the heterogeneity of NAFLD patients with respect to its primary drivers and co-existing disease modifiers and the great significance of this heterogeneity if put in new nomenclature to the discovery of highly effective drug treatments for this global disease. Second, an interesting point in this consensus is taking into account the psychological state of the patient as the suggested nomenclature MAFLD doesn't include words such as 'alcoholic' that is probably inserting the responsibility on the patient as having made their disease. Third, the consensus group suggests detailed phenotyping of MAFLD patients that will help to identify risky peoples, help to make preventive plans, and improvements in clinical trial strategy. Fourth, it's anticipated that, the noninvasive blood biomarkers or imaging techniques are in hop to replace liver biopsy for the diagnosis of disease. Because, the performance of scores such as (NFS) and
(FIB-4) may vary across the life span and the variation of transient elastography liver stiffness cut offs in different racial populations/subpopulations. So that, the consensus group recommends the factors that form the heterogeneity of NAFLD be thought of once designing and applying riskstratification scores and algorithms for NAFLD. This will maximize the benefit and clinical usefulness of these noninvasive biomarkers tools.

However, some questions remain to be answered; even after the suggested new acronym MAFLD that is more accurately and more descriptive to current knowledge of fatty liver diseases, we still need exclusion of other metabolic liver conditions most commonly associated with fatty liver disease such as drugs (e.g., amiodarone, tamoxifen,), metabolic abnormalities (e.g., galactosemia, glycogen storage diseases, and tyrosinemia), nutritional conditions (e.g., over nutrition, severe malnutrition, total parenteral nutrition, or even additional health troubles (e.g., celiac disease). Moreover, we need also to exclude other metabolic diseases which may aggravate the course of NAFLD, e.g., mutations for hemochromatosis. This means to some extent that, MAFLD may remain a disease of exclusion rather than inclusion. Second, the term metabolic needs more explanations and strict definitions to be more applicable in clinical practice and drug development.

\section{Acknowledgments}

Funding: The study was supported by Public Donation for ELRIAH.

\section{Footnote}

Provenance and Peer Review: This article was commissioned by the editorial office, Hepatobiliary Surgery and Nutrition. The article did not undergo external peer review.

Conflicts of Interest: Both authors have completed the ICMJE uniform disclosure form (available at https://hbsn. amegroups.com/article/view/10.21037/hbsn-20-438/coif). The authors have no conflicts of interest to declare.

Ethical Statement: The authors are accountable for all aspects of the work in ensuring that questions related to the accuracy or integrity of any part of the work are appropriately investigated and resolved.

Open Access Statement: This is an Open Access article 
distributed in accordance with the Creative Commons Attribution-NonCommercial-NoDerivs 4.0 International License (CC BY-NC-ND 4.0), which permits the noncommercial replication and distribution of the article with the strict proviso that no changes or edits are made and the original work is properly cited (including links to both the formal publication through the relevant DOI and the license). See: https://creativecommons.org/licenses/by-nc-nd/4.0/.

\section{References}

1. Kleiner DE, Makhlouf HR. Histology of Nonalcoholic Fatty Liver Disease and Nonalcoholic Steatohepatitis in Adults and Children. Clin Liver Dis 2016;20:293-312.

Cite this article as: Shiha G, Mousa N. Non-alcoholic steatohepatitis or metabolic-associated fatty liver: time to change. HepatoBiliary Surg Nutr 2021;10(1):123-125. doi: 10.21037/hbsn-20-438
2. Bellentani S, Tiribelli C. Is it time to change NAFLD and NASH nomenclature?. Lancet Gastroenterol Hepatol 2017;2:547-8.

3. Eslam M, Sanyal AJ, George J, et al. MAFLD: a consensusdriven proposed nomenclature for metabolic associated fatty liver disease. Gastroenterology 2020;158:1999-2014.e1.

4. Brunt EM, Ramrakhiani S, Cordes BG, et al. Concurrence of histologic features of steatohepatitis with other forms of chronic liver disease. Mod Pathol 2003;16:49-56.

5. Choi HSJ, Brouwer WP, Zanjir WMR, et al. Nonalcoholic Steatohepatitis Is Associated With Liver-Related Outcomes and All-Cause Mortality in Chronic Hepatitis B. Hepatology 2020;71:539-48. 